\title{
Implication of Intrinsic Intraocular Risk Factors at Ocular Blood Flow to Primary Glaucoma
}

\author{
Edward Ath Skoufis* and Konstantinos Diamantopoulos \\ Ophthalmology Department, Diagnostic and Therapeutic Center of Athens Hygeia, Greece
}

Received: 啙 January 24, 2018; Published: 制 February 08, 2018

*Corresponding author: Edward Ath Skoufis, Ophthalmology Department, Diagnostic And Therapeutic Center of Athens Hygeia, Er Stavrou 4, Marousi, Greece

\begin{abstract}
Purpose: To assess the implication of intrinsic intraocular risk factors at ocular blood flow (OBF) to primary glaucoma (POAG).

Method: In a retrospective control study included 51 consecutive patients, mean age 50, 5 \pm 7 , in follow-up 2008-2012 divided in control group (CG) 15 patients without POAG, group A (GRA) 15 patients with POAG, group B (GRB) 21 patients with POAG, 9 of them with disc hemorrhage (DH) and 12 with peripapillary atrophy (PPA). We performed baseline glaucoma examination including monitoring pulsatile ocular blood flow (POBFA), pulse amplitude (PA), central corneal thickness (CCT), OCT and visual field (VF) examination. Exclusion criteria: Previous ocular trauma and surgery, pathologies with evident VD. Paired T-test and linear regressions used for statistical analysis.
\end{abstract}

Results: Our findings indicated a strong correlation between OBFA, IOP and an interrelation between POAG and intrinsic risk factors, $\mathrm{DH}$ and PPA $(\mathrm{P}=0,001)$. In GRB versus $\mathrm{CG}$,GRA POBF were very sensible and fluctuated depending on the intraocular VD.CCT and IOP changes $(\mathrm{P}=0,002)$, showed the abnormality auto regulation( $\mathrm{r}=0,650)$. In GRB the POBF and PA were lower $\geq 8 \%$ than other subgroups $(\mathrm{P}<0,001)$. LATANOPROST with IOP reduction from baseline $30,8 \%$, POBF improved $16,6 \%$, PA $10 \%$ was the best therapy in GRA. In GRB the switch therapy DORZOLAMIDE + LATANOPROST, DORZOLAMIDE + BIMATOPROST IOP reduction 33.0\% from baseline, POBF improved 36\%, PA 22\%, with added therapy NILVADIPIN 60 mgr + GINKGO BILOBA $150 \mathrm{mg}$, VISIONACE plus the hemodynamic parameters further improved $\geq 5 \%$.

Keywords: Vascular Dysregulation; pulsatile ocular blood flow; Pulse Amplitude; Factor Risk , Disc Hemorrhage; Peripapillary Atrophy; Primary Glaucoma; Hemodynamic Parameters; Selective Therapy.

Abbreviations: VDR: Vascular Dysregulation; POAG: Primary Glaucoma; POBF: Pusatile Ocular Blood Flow; PA: Pulse Amplitude; DH: Disc Hemorrhage; PPA: Peripapillary Atrophy; CCT: Central Corneal Thickness; VF: Visual Field

\section{Introduction}

Glaucoma is multifactorial disease [1-3]. Investigations of vascular theory in Glaucoma, changed the treatment strategy [4,5]. Vascular dysregulation (VDR) as a systemic dysfunction develop more efficient treatment approach $[4,6]$ compound by extra ocular and intraocular factors [6,7]. DH and PPA are intrinsic important expressions of VDR [8].

Purpose: To assess the implication of intrinsic intraocular risk factors at ocular blood flow (OBF) to primary glaucoma (POAG) patients evaluating medical strategy.

\section{Method}

In a retrospective control study included 51 consecutive patients, mean age 50,5 \pm , in follow-up 2008-2012 divided in control group (CG) 15 patients without POAG, group A(GRA) 15 patients with POAG, no significant vascular dysregulation(VDR) factor, group B (GRB) 21 patients with POAG, 9 of them with disc hemorrhage (DH), identified supratemporal in 5 cases, infratemporal 4 cases, and 12 with peripapillary atrophy (PPA) was measured in three sectors around the disc circumference 1-5 o'clock. We performed standardized protocol of baseline glaucoma 
examination including monitoring pulsatile ocular blood flow (POBFA), pulse amplitude (PA), central corneal thickness (CCT), OCT, and visual field (VF) examination. Exclusion criteria: Previous ocular trauma and surgery, pathologies with evident VD. Paired T-test and linear regressions used for statistical analysis.

\section{Results}

Our findings indicated a strong correlation between OBFA, IOP and an interrelation between POAG and intrinsic risk factors, DH and PPA $(\mathrm{P}=0,001)$. In GRB versus CG,GRA POBF were very sensible and fluctuated depending on the intraocular VD.CCT and IOP changes $(\mathrm{P}=0,002)$, showed the abnormality auto regulation $(\mathrm{r}=0,650)$. GRB showed a mean decrease of $\mathrm{OBF}=26$, $5 \%, \mathrm{PA}=28,5 \%$ from control group and $\mathrm{OBF}$ and $\mathrm{PA}$ were lower $\geq 10 \%$ than GRA $(\mathrm{P}<0,001)$, explained by correlated vascular risk factors. OCT shows a significant difference between CG and eyes with PPA ( $p=0,0005)$, which remained unchanged. LATANOPROST with IOP reduction from baseline 30, 8\%, POBF improved 16, 6\%, PA $10 \%$ was the best therapy in GRA. In GRB the switch therapy DORZOLAMIDE + LATANOPROST, DORZOLAMIDE + BIMATOPROST IOP reduction $33.0 \%$ from baseline, POBF improved 36\%, PA $22 \%$, with added therapy NILVADIPIN $60 \mathrm{mgr}+$ GINKGO BILOBA $150 \mathrm{mg}$, VISIONACE plus the hemodynamic parameters further improved $\geq 10 \%$.

\section{Discussion}

These findings indicated a multifactorial VDR not only "extrinsic" but also intrinsic $[9,10]$. DH, PPA must be considered risk factor of glaucoma progression [9]. DH theorized that they are as result of a micro vascular occlusion of the disc blood supply or by optic nerve neurodegenerative [11,12]. PPA describes as atrophy or thinning of retinal layers and retinal pigment epithelium $[2,13]$. In GRB the circadian fluctuations of OBF with PA reduction are significant predictor of glaucoma progression, reflected to $\mathrm{VF}$ and OCT. Our selective therapy is to get IOP as low as possible with important OBF improved [14-16].

\section{Conclusion}

DH and PPA must be considered risk factor of glaucoma progression and intrinsic important expressions of VDR. Ocular hemodynamic parameters are compromised by DH and PPA in glaucomatous eyes. Fluctuations of $\mathrm{OBF}$ and $\mathrm{PA}$ reduction are indices of vascular dysregulations. Careful observations and multimodal therapy are necessary $[10,13,15]$.

\section{References}

1. G Lambrou, E Greve (1989) Ocular Blood Flow, pp: 205-231.

2. JB Jonas, MC Fernandez (1992) Glaucomatous parapapillary atrophy Occurrence and Correlations. Arch Ophthalmol 110(2): 214-222.

3. L Bonomi, G Marchini, M Marraffa (2000) vascular risk factors for POAG Ophthalmology 107(7): 1287-1293.

4. A Harris (2001) Changing perspectives in Glaucoma therapy. Int Glaucoma Symposium, Milan, Italy.

5. ED Ath Skoufis, N Michalopoulos (2017) Interference of independent risk factors important integral part of OBP in latent Glaucoma and efficient treatment. EC Ophthalmology 6(6): 157-160.

6. J Flammer, P Costa (2002) the impact of ocular blood flow in Glaucoma. Progress in Retinal and Eye Research 21(4): 359-393.

7. SS Hayreh (1994) Progress in the understanding of the vascular etiology of Glaucoma. Current Opinion in Ophthalmology 5(2): 26- 30.

8. ED Ath Skoufis, S Georgaras (2008) Validation of ocular perfusion changes in NTG and their confrontation. $8^{\text {th }}$ European Glaucoma Congress, Berlin, Germany.

9. ED Ath Skoufis, S Georgaras (2007) Assessment and treatment of Ocular Blood Flow fluctuations in Myopic Glaucomatous Eyes. $6^{\text {th }}$ International Glaucoma Symposium Athens, Greece.

10. ED Ath Skoufis, E Tsichlis (2010) Diagnostic compatibility of structural and hemodynamic parameters in preperimetric glaucoma. $1^{\text {st }}$ World Congress on Controversies in Ophthalmology, Prague, Czech Republic.

11. N Orzalesi, L Rossetti (2007) Vascular risk factors in Glaucoma. Graefe's Arch Clin Exp Ophth 245(6): 795 - 802.

12. M Cristina Leske, Suh Yuh Wu, Anselm Hennis (2008) Risk factors for incident OAG. Barbados Eye Studies Ophth 115(1): 85-93.

13. R Oh Eineachain (2011) Glaucoma in Focus. Eurotimes 16(6): 4.

14.ED Ath Skoufis (2011) Vascular Dysregulation and lifestyle important part of ocular blood perfusion. Joint Congress of SOE-AAO, Geneva, Switzerland.

15. ED Ath Skoufis, I Segos (2012) Antiaging Therapy on OBF in Glaucoma. European Glaucoma Congress, Copenhagen, Denmark.

16. ED Ath Skoufis (2017) Contemporaneus concept of Antiaging therapy as valuable paradigm on OBF enhancement in Glaucoma. Advances in Ophthalmology \& Visual System 6(3). (c) This work is licensed under Creative

To Submit Your Article Click Here: Submit Article

DOI: $10.32474 /$ TOOAJ.2018.01.000105

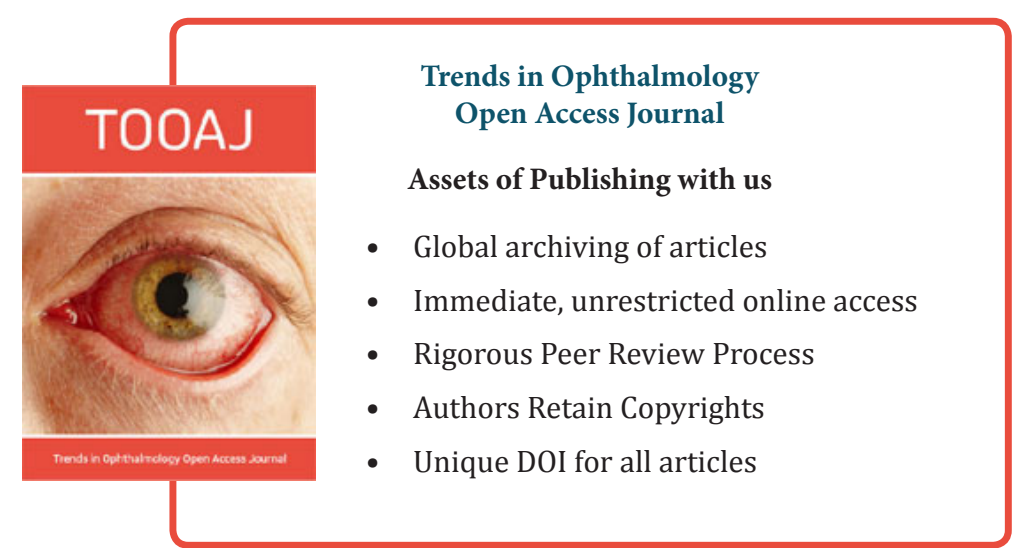

